\title{
Curation of a Multiple Myeloma Cell Surface Database for Immunotherapy Development Emily Adaniya ${ }^{1}$, Christina $\mathbf{Y u}^{2}$, Fabiana Perna ${ }^{2}$ \\ ${ }^{1}$ Indiana University School of Medicine; ${ }^{2}$ Indiana School of Medicine, Department of Medicine
}

Background/Objective: Every year in the United States, 32,000 individuals are diagnosed with Multiple Myeloma (MM) and 13,000 die from the disease. MM is a cancer of the plasma cells and while treatment exists, MM remains incurable. Our overall goal is to identify biologically and therapeutically relevant cell surface targets to develop MM immunotherapy.

Methods: Given the genomic heterogeneity of MM, we performed surface analysis of seven MM cell lines by Mass-Spectrometry (MS), generating a pool of candidate proteins. Lacking specific tools for studying cell surface proteins, we developed an integrated computational tool with five distinct databases, and scored the likelihood of cell surface location for each molecule. One point was given for each database a protein appeared in. A protein receiving a score of three or more was considered a cell surface protein with high confidence. However, about one third of the candidates were not detected by the computational tool, thus requiring manual curation. We used UniProt and GeneCards to determine the subcellular locations of the unannotated IDs, and further confirmed their location with the computational tool.

Results: Of the 5,454 UniProt IDs produced by MS, 2,026 were not annotated. Causes of an unannotated ID included having an obsolete ID, being an isoform, and/or not being located on the cell surface. Through manual annotation, 8 unique cell surface IDs were added to the existing 448. The 456 targets were further analyzed based on their expression profiles in both $\mathrm{MM}$ and normal tissues resulting in 94 of the most promising targets, some of which would have been missed without manual curation.

Conclusion/Potential Impact: Public genomic databases are often incomplete and may contain errors making manual curation a necessary step in error reduction. Left unchecked, promising surface targets could have been overlooked hindering the creation of potentially curative immunotherapies. 